\title{
Wide-Area Backup Protection and Protection Performance Analysis Scheme Using PMU Data
}

\author{
Fangzhu Yu, Campbell Booth, Adam Dysko and Qiteng Hong \\ Department of Electronic and Electrical Engineering, University of Strathclyde, Glasgow, UK \\ e-mail: fangzhu.yu@strath.ac.uk \\ tel: $+44(0) 1414447355$
}

\begin{abstract}
This paper presents a wide-area backup protection scheme that incorporates protection performance analysis based solely on voltage from Phasor Measurement Units (PMUs). The system reports and summarizes information relating to fault detection, and identification of the faulted circuit(s) protection/circuit breaker operation (i.e. whether it is correct or not), in a short period of time. It can also be applied as an effective and relatively simple, fast, wide-area backup protection to improve the resilience of power systems. Case studies are presented, where the proposed wide-area backup protection scheme is validated using the IEEE 14-bus network. It is demonstrated that the proposed scheme is capable of correctly detecting faults (including high-resistance faults) in less than 100 $\mathrm{ms}$ from fault inception and can report on whether the protection/circuit breakers have operated as expected within a further 100 $\mathrm{ms}$, thereby coordinating with existing protection systems with a view to enhancing the system reliability and security by appending existing protection systems with system-wide information. Applicability of the developed system to large-scale power systems is also demonstrated. Discussion relating to how this method can be cost effective through exploitation of existing PMU data, which may already be used for other purposes, is included.
\end{abstract}

Keywords: Backup protection, voltage-based fault detection, system simplification, wide-area protection, PMU application

\section{Introduction}

Backup protection plays a key role in isolating faults in the events of failure in main protection systems, thus offering an extra layer of assurance for overall power system reliability. However, if the backup protection does not operate correctly, it can also cause significant disruption to power supply. Presently, for all feeders and interconnectors at transmission levels, the main protection is typically provided by at least two independent discriminative high-speed protection systems (i.e. distance and /or line differential protection), the outputs of which are selectively allocated to independent tripping systems with separate D.C. supplies [1]. The backup protection is typically provided by distance (e.g. zone 2 and 3), overcurrent and earth fault protection (typically with IDMT characteristics). It has been noted that the root causes of a number of historical blackout events were due to transmission backup protection not operating correctly [2-4], particularly with reference to zone 3 of distance protection schemes. The increasing penetration of renewables could lead to a significant reduction and increased variability in fault levels. These changes in fault levels could introduce further challenges to the backup zones of distance and overcurrent protection, either rendering them prone to unwanted or nonoperation or requiring complex evaluation and possibly adaption of settings based on prevailing system conditions [5].

The application of wide area measurements from PMUs for backup protection is considered as an effective extra layer to conventional backup protection [6]. PMUs and digital substation technologies has seen a growing popularity and led to increasing development of Wide-Area Monitoring, Protection and Control (WAMPAC) functions [7]. PMUs can provide synchronously measured data with high reporting rates (up to 200 $\mathrm{Hz})[8,9]$ to implement a variety of WAMPAC applications. Currently, PMU protection applications mostly focus on backup (often requiring current measurements), adaptation of protection settings and/or protection blocking in certain cases to enhance security [10-13]. Communication system delays associated with wide-area measurements, in most cases, are too long for main short-circuit protection applications [6, 12]. However, such measurements are adequate for wide-area monitoring and backup protection schemes [14].

A wide range of wide-area backup protection (WABP) schemes have been proposed and reported in [15-23]. The key shortcoming of these systems is that they often require a relatively high number of measurements and related data, including both voltage and current and/or status of circuit breakers. As a result, these schemes can be both expensive and complex when considering the practical implementation in a wide area. Furthermore, the need for both voltage and current measurements at every feeder is likely to require relatively high processing power, high resolution of data (e.g. sample-by-sample current measurements), as well as adequate communication bandwidth and infrastructure. There are three main categories of methods reported in the literature concerning wide area fault detection and backup protection: current differential based schemes applied to wide areas for backup; impedance-based methods using wide-area measurements of both voltage and current; other 
methods with requirements to gather the status of protective relay devices. Current differential based methods, as reported in $[16,17]$, can accurately detect faults and are immune to nonfault transients such as load swings and temporary overloads. However, such schemes require high volumes of measured data and notably higher communication system performance than the method reported in this paper. Impedance based methods reported in [18-20] require both voltage and current measurement, and they may be difficult to coordinate and could be prone to issues associated with decreasing and variable system strength (e.g. affecting remote infeed levels). Accordingly, the zone reach accuracy of distance protection, especially for backup zones (i.e. zone 2 and zone 3 ), could be compromised. These issues will be more apparent with increasing penetration of renewables [5]. A number of other methods, which require information relating to the status of protection relay devices, are proposed [6, 15, 21, 22]. These have purported advantages in terms of simplicity, security and dependability. However, they often require both current and voltage measurements, in addition to circuit breaker status data, potentially rendering them impractical and costly.

To address the aforementioned shortcomings of the exiting backup protection schemes, this paper presents a new wide-area backup protection and protection performance analysis scheme that only requires voltage measurements from the busbars (as opposed to individual terminals). The proposed system is capable of providing relatively fast and accurate fault location to the circuit/feeder level, followed by the analysis of protection and circuit breaker performance from observed voltage magnitudes and ability to provide backup protection for the network. It has the following compelling benefits:

1. Fault detection, faulted line identification, and subsequent protection/circuit breaker performance analysis.

2. Cost-effective compared to the aforementioned other wide-area backup protection schemes. The proposed scheme only requires PMUs to be installed at the bus bar level, as opposed to many other reported systems, which require PMUs to be available at each feeder, thus requiring additional measurement transformers (and measurements of current in addition to voltage) and possibly higher communications bandwidth and measurement resolution.

3. The system operates for faults at any location on the lines - even very "close up" faults have no detrimental impact on the proposed scheme, whereas impedance-based schemes may be compromised in such situations.

4. The configuration and settings of the proposed scheme can be readily and automatically recalculated if there is a significant change in the power system structure and/or fault levels, thus being highly adaptable to system operating conditions.

5. The proposed scheme can operate relatively fast, compared to Zone 2 distance protection and overcurrent protection, due to the accurate identification of faulted feeder - no need for long time delay for discrimination.

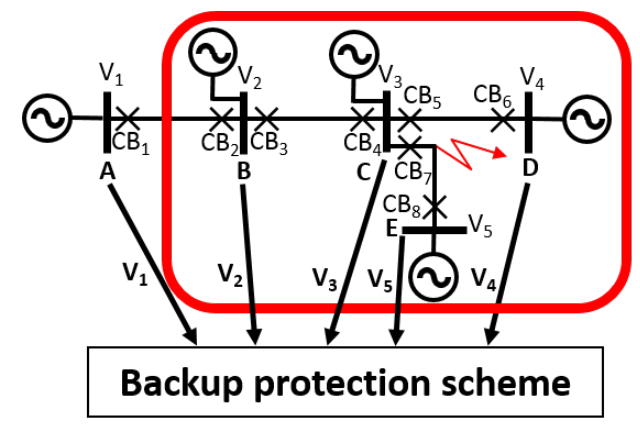

Figure 1: Single line diagram of a 5-node system

The remainder of the paper is structured as follows: Section 2 presents the design and operating principles of the proposed wide-area backup protection scheme; Section 3 discusses the setting of the proposed scheme; In Section 4, case studies using the IEEE 14-node system to validate the performance of the scheme is presented; the applicability of the system to largescale power systems is discussed in Section 5; and conclusions and future work are presented in Section 6.

\section{Principle of Scheme Operation}

\subsection{Overview of the proposed scheme}

In power systems, short circuit faults will typically lead to voltage depressions measured at substations in the vicinity of the fault. Using voltage measurement data supplied from remote PMUs, the proposed scheme detects the presence of a fault, identifies any subsequent status changes of circuit breakers solely from analysis of the measured voltage profile and identifies the faulted feeder and any failure of protection or circuit breaker(s). Since the time required for analysis is short, backup protection can also be provided with this information.

The proposed scheme consists of three main stages of operation: fault region identification; faulted feeder identification; and backup protection based on the analysis of circuit breaker status. The first stage is initiated if at least one voltage measurement from any of the PMUs reduces below a certain threshold, and it will generate a network region with multiple candidate lines and nodes within which the fault occurs. The second stage is based on the analysis of stage one outputs and the characteristics of node voltage magnitudes to further locate the faulted element. Based on the identified faulted element in the second stage, the performance of the main protection and the operation of the associated circuit breakers are analysed in the third stage, which then enables the backup protection action. The detailed operational principles of the three main stages are presented in the following.

\subsection{Stage one - fault region identification}

In order to clearly explain the concept of the scheme, an example of a 5-node radial system with a three-phase solid fault close to node $\mathrm{C}$, on the line connecting $\mathrm{C}$ and $\mathrm{E}$, as shown in Fig. 1, will be used. 
In stage one, the scheme monitors the voltage magnitudes at all nodes via the PMUs and records the steady-state voltage magnitudes of all nodes. The pre-fault voltage magnitude of node $i$ is denoted as $V_{0}^{i}$, which belongs to a voltage set $\boldsymbol{V}_{\mathbf{o}}$ that contains pre-fault voltage magnitudes from all nodes. A voltage depression will be observed at several locations around the vicinity of a fault when it occurs and the operation of the scheme is initiated by a pre-defined voltage threshold (the threshold is a relative value to $V_{0}^{i}$ ). This is explained further in Section 3.2. For the scheme, determinations are based on the ratio of monitored voltage magnitudes of all nodes to their pre-fault values $V_{0}^{i}$, which are denoted as $k_{i}$, i.e. $k_{i}=V_{i} / V_{0}^{i}$, and the set containing $k_{i}$ from all nodes is denoted as $\boldsymbol{K}$. If $k_{i}$ of at least one node is lower than the threshold, the node with the smallest $k_{i}$ (i.e. $k_{\text {min }}$ ), defined as $N_{\min }$, and is identified as a node that is definitely connected to the faulted feeder. The voltage magnitude of $N_{\min }$ is defined as $V_{\min }$. A subset of nodes is then formed, which includes $N_{\min }$ and all neighbouring nodes. This subset of nodes, $\boldsymbol{N}_{\boldsymbol{r e g}}$, defines the region of the network where the fault is located. The corresponding subset of $\boldsymbol{K}$, denoted as $\boldsymbol{K}_{\boldsymbol{r e g}}$, contains the $k_{i}$ of all nodes within $\boldsymbol{N}_{\boldsymbol{r e g}}$. The set contains the voltage magnitudes of all nodes within $\boldsymbol{N}_{\boldsymbol{r e g}}$ is denoted as $\boldsymbol{V}_{\boldsymbol{r e g}}$.

For the scenario shown in Fig. 1, $V_{0}^{i}$ of all nodes is 1 p.u. and for this fault, $V_{3}$ would have the largest voltage depression. The voltage magnitudes (as well as $k_{i}$ ) of the neighbouring nodes $\left(V_{2}, V_{4}\right.$ and $\left.V_{5}\right)$ will depend on line lengths, the fault levels within the system, the topology of the system and the fault resistance. The impact of these factors is described further in Section 3. The fault region can be identified as the area bounded by nodes B, C, D and E. It is known that C is definitely connected to the faulted feeders, and that the other node of the faulted feeder must be within $\boldsymbol{N}_{\boldsymbol{r e g}}$. That is, one of nodes B, D or E.

\subsection{Stage two - faulted feeder identification}

The process of faulted feeder identification in stage two is illustrated in Fig. 2, where it can be seen that the node set $\boldsymbol{N}_{\boldsymbol{r e g}}$, the corresponding $\boldsymbol{K}_{\boldsymbol{r e g}}$ set and $\boldsymbol{V}_{\boldsymbol{r e g}}$ set established in stage one are used as the main inputs to stage two. If there are more than two nodes in $\boldsymbol{N}_{\text {reg }}$, then identification of the faulted circuit requires operation of one of the circuit breakers and a consequent observable voltage magnitude change at a number of PMUs. The assumption that at least one line-end primary protection operates correctly is resumed reasonable due to the redundancy of two independent discriminative high-speed main protection schemes and the communication channels, and often the use of different types of protection schemes in terms of principles. The probability that all communication channels fail at the same time is extremely low. The time period $t$, shown in Fig. 2, is pre-defined and can be configured based upon the anticipated maximum main protection fault clearance time (e.g. $140 \mathrm{~ms}$ in Great Britain [5]). The measured node voltages are examined to identify any instances of voltage recovery, i.e. either partial recovery due to the opening of one of the circuit breakers only, or full recovery (restoration of the voltage to normal operational range), following the successful fault clearance

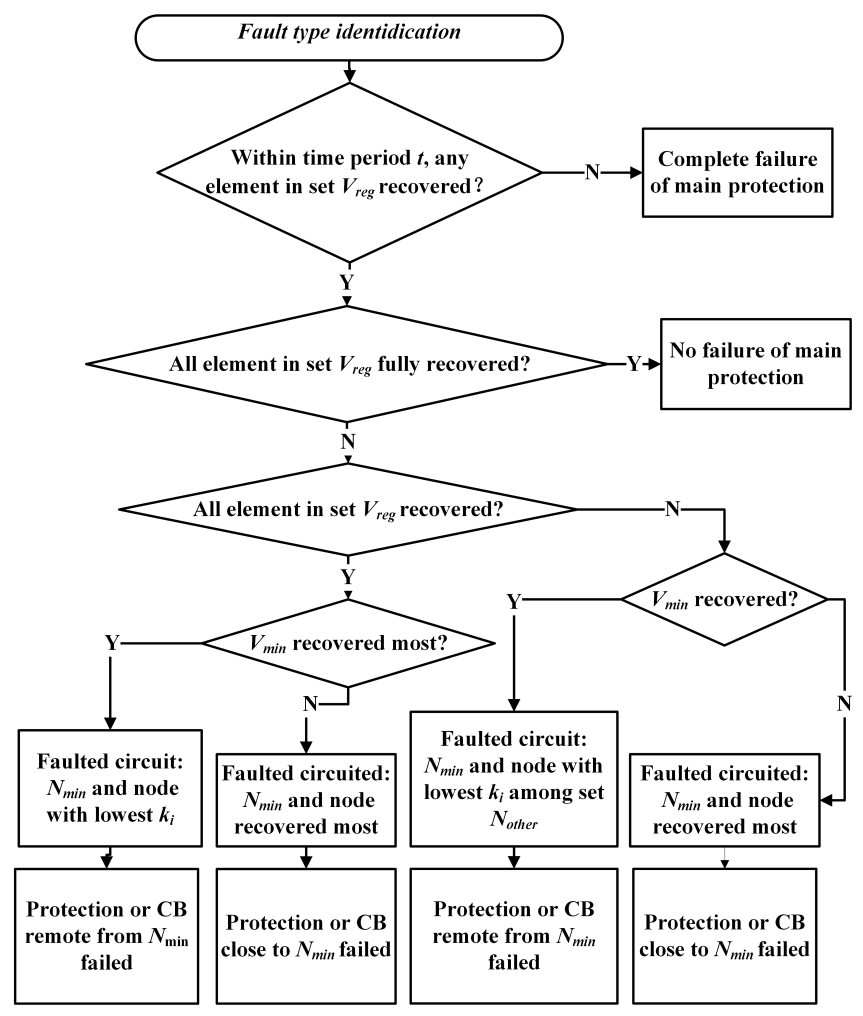

Figure 2: Flow chart of stage two-faulted feeder identification

at all terminals of the faulted feeder. If all voltages are fully recovered during time $t$, then all protections/breakers have operated correctly and no further analysis is required. Conversely, if there is no voltage recovery observed within time $t$, then all main protections would have failed at all line terminals (which is highly unlikely) and the scheme can report upon the faulted region (potentially containing several feeders) based on stage one. In this stage, it is presumed that the main protection has operated correctly at least at one end of the faulted feeder (the probability of failure of both main protections at all terminals of the line is assumed to be negligible).

With operation of line terminal protection schemes and their controlled circuit breakers, there are four possible behaviour patterns of the voltages within set $\boldsymbol{V}_{\boldsymbol{r e g}}$ that may be observed. 1) the voltage could remain unchanged; 2) it could reduce further; 3) it could partially recover (e.g. by less than $90 \%$ of the total voltage dip magnitude), or 4) fully recover (e.g. to greater than $90 \%$ of the total voltage dip magnitude). The value of $90 \%$ to signify full recovery is deemed acceptable. These values have been proposed based on the results of extensive simulations and can be modified if necessary. Fig. 3 shows the voltage behaviours for the fault scenario depicted in Fig. 1. The variable $x$ indicates the maximum magnitude of the voltage dips and $y$ indicates any increase in voltages after the voltages have reached their minimum values. A dedicated functional block has been developed for capturing the values of $x$ and $y$ during simulation. The fault is applied at $0.5 \mathrm{~s}$ in the simulation and it is assumed that $C B_{8}$ at node $C$ operates correctly and opens 80 $\mathrm{ms}$ later at $0.58 \mathrm{~s}$. A positive value of the ratio of $y$ to $x$ is used to identify voltage recovery (which could be indicative of either partial or full recovery). A ratio of $y$ to $x$ of greater than 0.9 in- 
dicates full recovery - e.g. in $V_{5}$ in Fig. 3. A negative value for $\mathrm{y}$ indicates a further depression after the initial depression (e.g. $V_{1}, V_{2}, V_{3}$ and $V_{4}$ ).

It is very important to identify when a voltage has partially or fully recovered (indicating that a circuit breaker has opened and therefore protection has operated), and when it remains unchanged or reduced further due to the operation of a circuit breaker remote from the measured voltage location. These behaviour patterns provide indication for further analysis of circuit breaker status and faulted feeders.

During and following fault events that are not properly responded to correctly (i.e. a line terminal protection system or its controlled breaker does not operate), realistically, only two scenarios can be observed. Scenario 1 is where all elements in set $\boldsymbol{V}_{\text {reg }}$ recover, but not all fully recover; while scenario 2 is where only some elements of set $\boldsymbol{V}_{\boldsymbol{r e g}}$ recover while the remaining voltages remain unchanged or decrease. A new set of elements within set $\boldsymbol{N}_{\text {reg }}$, from stage 1 containing all voltages that subsequently exhibit partial recovery or full recovery behaviour is denoted as set $\boldsymbol{N}_{\boldsymbol{u}}$. The remaining elements within the set $\boldsymbol{N}_{\text {reg }}$ which have not partially recovered or fully recovered form the set $\boldsymbol{N}_{\text {other }}$.

In scenario 1 , if $V_{\min }$ has not recovered most significantly within set $\boldsymbol{V}_{\boldsymbol{r} e \boldsymbol{g}}$, then the faulted circuit is identified as the feeder connected between $N_{\min }$ and the node with the most significant voltage recovery within set $\boldsymbol{N}_{\text {reg }}$. Operational failure of the protection/circuit breaker on this feeder at $N_{\min }$ can be reported. Otherwise, if $V_{\text {min }}$ has recovered most significantly within the set $\boldsymbol{V}_{\text {reg }}$, then the faulted circuit is identified as the feeder connecting $N_{\min }$ and the node with the lowest voltage magnitude within set $\boldsymbol{N}_{\text {reg }}$. Operational failure of the protection/circuit breaker on this feeder remote from $N_{\min }$ can be reported.

In scenario 2 , if $V_{\min }$ has recovered, the faulted circuit is identified as the feeder connected between $N_{\min }$ and the node with the lowest voltage magnitude within $\boldsymbol{V}_{\text {other }}$. Operational failure of the protection/circuit breaker at the node on this feeder remote from $N_{\min }$ can be reported. Otherwise, the faulted circuit is identified as the feeder within $N_{\min }$ and the node with the most significant voltage recovery within set $\boldsymbol{N}_{\boldsymbol{u}}$. Operational failure of the protection/circuit breaker on this feeder at $N_{\text {min }}$ can be reported.

For the scenario depicted in Fig. 1, the resulting voltage profiles are presented in Fig. 3. C is $N_{\min }$ and, at the initial stage, it can be deduced that the other node connected to the faulted feeder is one of either nodes B, D or E. From around $0.6 \mathrm{~s}$, there is no recovery behaviour for $V_{\min }\left(V_{3}\right)$, but $V_{5}$ has the most significant recovery (fully recovering to nominal from $0.64 \mathrm{~s}$ ). Accordingly, the faulted circuit is then identified as the feeder connected between $\mathrm{C}$ and $\mathrm{E}$. However, the protection or circuit breaker at $\mathrm{C}\left(C B_{7}\right)$ has failed as the voltage remains depressed after the opening of the circuit breaker at $\mathrm{E}$ - note that all other voltages also remain depressed and do not recover - in this case backup protection would be required to operate. The system would quickly report on the failure of the protection/circuit breaker at $\mathrm{C}$.

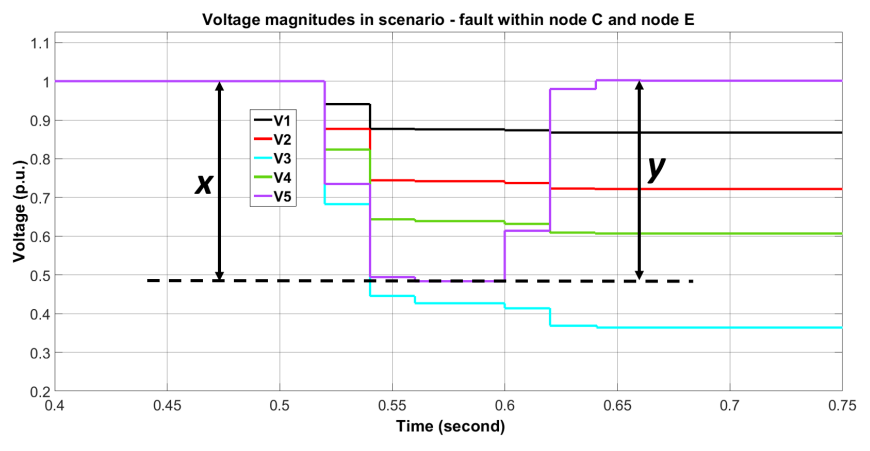

Figure 3: Voltage magnitudes of 5-bus system

\subsection{Stage three - backup protection}

With the analysis of the results from stage two - faulted circuit identification and main protection performance analysis, backup protection can then be applied.

The scheme will send tripping signals to all closed neighbouring (directly connect to the same node) circuit breakers around the failed circuit breaker to provide backup protection. For the 5-node case study shown in Fig. 1, the faulted circuit is identified as the line connecting nodes $\mathrm{C}$ and $\mathrm{E}$ and the protection/circuit breaker at $\mathrm{C}$ is identified as having failed to operate from stage two of the scheme operation. A trip signal will be sent to $C B_{4}, C B_{5}$ and $C B_{7}$ to disconnect the faulted circuit from the system to provide backup protection.

\subsection{Operation of unbalanced faults}

Similar principles with detection of three-phase faults, other types of faults can be detected by analysis of negative sequence components (phase-phase faults) and zero sequence components (unbalanced earth faults). Phase-phase faults will introduce negative sequence components apart from positive sequence. Similarly, unbalanced earth faults will introduce zero sequence components to the circuit. Negative sequence and zero sequence voltages are used to identify unbalanced faults in the scheme, since pre-fault values for negative and zero sequence voltages would normally be zero and will increase from zero to above zero due to phase-phase faults or unbalanced earth faults, which is more sensitive than positive sequence [24].

To demonstrate the operation of the scheme for unbalanced earth faults, a single-phase to earth fault is applied close to node $\mathrm{C}$, on the line connecting $\mathrm{C}$ and $\mathrm{E}$ in the 5-node radial system as shown in Fig.1.

For unbalanced earth faults (single-phase to earth or phasephase to earth faults), stage one can identify the occurrence of faults and the corresponding fault region as presented in Fig.4. Zero sequence voltage magnitudes are monitored and recorded at all nodes in stage one. The meanings and characteristics of variables in this scenario are similar to three-phase fault scenarios but have different denotations to distinguish. The zero sequence voltage magnitudes of node $i$ is denoted as $V_{0 i}$, which belongs to a voltage set $\boldsymbol{V}_{\mathbf{o}}$. The pre-fault zero sequence voltage magnitude of node $i$ is denoted as $V_{00}^{i}$, which belongs to a voltage set $\boldsymbol{V}_{\text {oo }}$ that contains pre-fault zero sequence voltage magnitudes from all nodes. Similar to three-phase fault identification process, for each cycle of fault identification, $V_{00}^{i}$ 


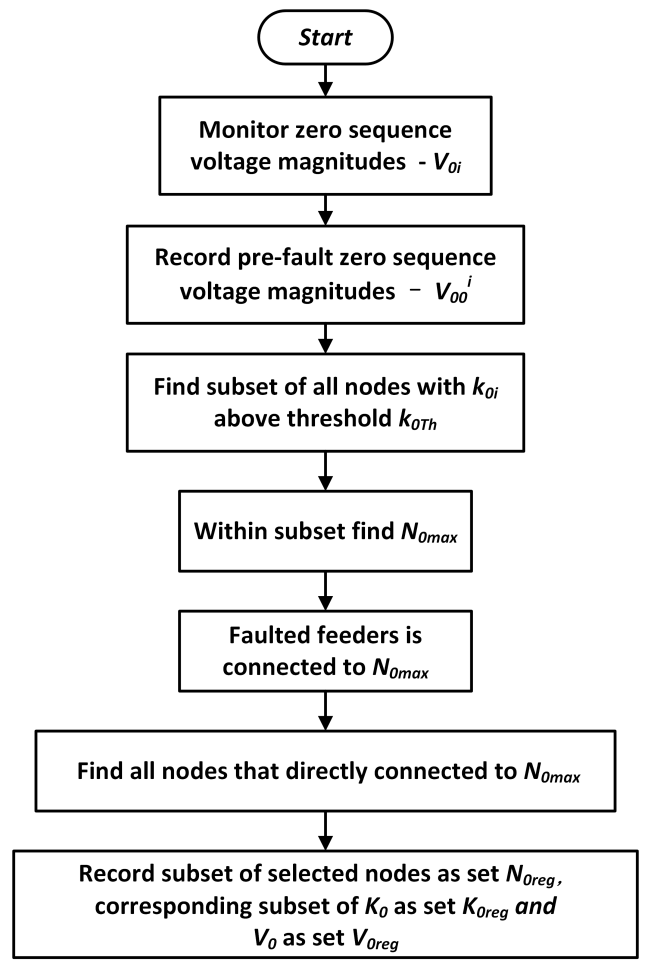

Figure 4: Flow chart of stage one for unbalanced earth faults - fault region

varies with time but fixed before occurrence of a fault. A rise of zero sequence voltage magnitudes will be observed at several locations around the vicinity of a fault when it occurs and the operation of the scheme is initiated by a pre-defined voltage threshold $k_{0 T h}$. the decisions are made based on the difference between monitored zero sequence voltage magnitudes of all nodes to their pre-fault values $V_{00}^{i}$, which are denoted as $k_{0 i}$, i.e. $k_{0 i}=V_{0 i}-V_{00}^{i}$, and the set containing $k_{0 i}$ from all nodes is denoted as $\boldsymbol{K}_{\mathbf{o}}$. Set $\boldsymbol{K}_{\mathbf{o}}$ is introduced to easily compare the rise of voltage instead of the absolute value of zero sequence voltage, which would be more flexible when pre-fault voltages have offsets. Before occurrence of an unbalanced earth fault, $k_{0 i}$ is always around 0 . With occurrence of an unbalanced earth fault, $k_{0 i}$ of node $i$ around the fault, will increase. If $k_{0 i}$ of at least one node is above the threshold, the node with the largest $k_{0 i}$ (i.e. $\left.k_{0 \max }\right)$, defined as $N_{0 \max }$, and is identified as a node that is definitely connected to the faulted feeder. The voltage magnitude of $N_{0 \max }$ is denoted as $V_{0 \max }$. Based on topology of the network, a subset of nodes is then formed, which includes $N_{0 \max }$ and all neighbouring nodes. This subset of nodes, $\boldsymbol{N}_{\text {oreg }}$, defines the region of the network where the fault is located. The corresponding subset of $\boldsymbol{K}_{\mathbf{o}}$, denoted as $\boldsymbol{K}_{\mathbf{o} \text { reg }}$, contains the $k_{0 i}$ of all nodes within $\boldsymbol{N}_{\mathbf{o r e g}}$. The corresponding subset of $\boldsymbol{V} \mathbf{o}$, denoted as $\boldsymbol{N}_{\text {oreg }}$, contains the $V_{0 i}$ of all nodes within $\boldsymbol{N}_{\text {oreg }}$. Same as three-phase fault scenario, all voltages $\left(V_{0 i}\right)$ are relative values to nominal voltages and expressed in p.u.

For the scenario shown in Fig.1, $V_{0 i}$ of all nodes is 0 p.u. before fault occurrence and with a single-phase to earth solid fault, $k_{03}\left(V_{03}\right)$ would have the largest rise. The fault region can be identified as the area bounded by nodes B, C, D and E. It is known that $\mathrm{C}$ is definitely connected to the faulted feeders, and

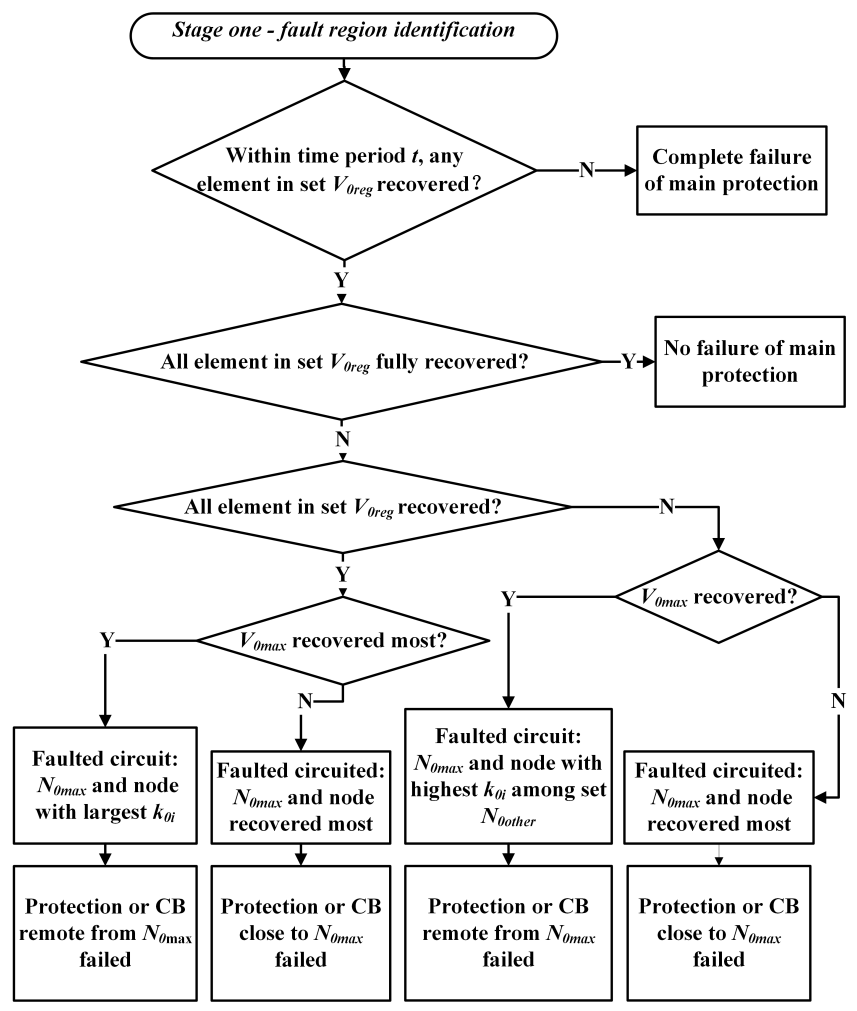

Figure 5: Flow chart of stage two for unbalanced earth faults - faulted feeder identification

that the other node of the faulted feeder must be within $N_{\text {oreg }}$, which is one of nodes B, D or E.

Under the assumption that the primary protection has operated correctly at least one end of the faulted feeder, the operation of stage two is illustrated in Fig.5.

If there is at least one node in $V_{\text {oreg }}$, then identification of the faulted circuit requires operation of one of the circuit breakers and a consequent observable zero sequence voltage magnitude $V_{0 i}$ change at a number of PMUs. The time period $t$, shown in Fig.5, is the same as the one defined for three-phase fault identification, which is discussed in Section2.3. The measured node zero sequence voltage magnitudes $V_{0 i}$ are examined to identify any instances of zero sequence voltage recovery, i.e. either partial recovery due to the opening of one of the circuit breakers only, or full recovery (falling back to the pre-fault voltage level), following the successful fault clearance at all terminals of the faulted feeder. If all voltages are fully recovered during time $t$, then all protections/breakers have operated correctly and no further analysis is required. Conversely, if there is no voltage recovery observed within time $t$, then all primary protections would have failed at all line terminals (which is highly unlikely) and the scheme can only report upon the fault region (potentially containing several feeders).

With operation of line-terminal protection schemes and their controlled circuit breakers, there are four possible behaviour patterns of the elements within set $\boldsymbol{V}_{\mathbf{o r e g}}$ that may be observed. 1) it could remain unchanged; 2) it could further increase 3) it could partially recover, or 4) fully recover (e.g. falling back with greater than $90 \%$ of the total voltage rise magnitude). The value of $90 \%$ to signify full recovery is deemed 


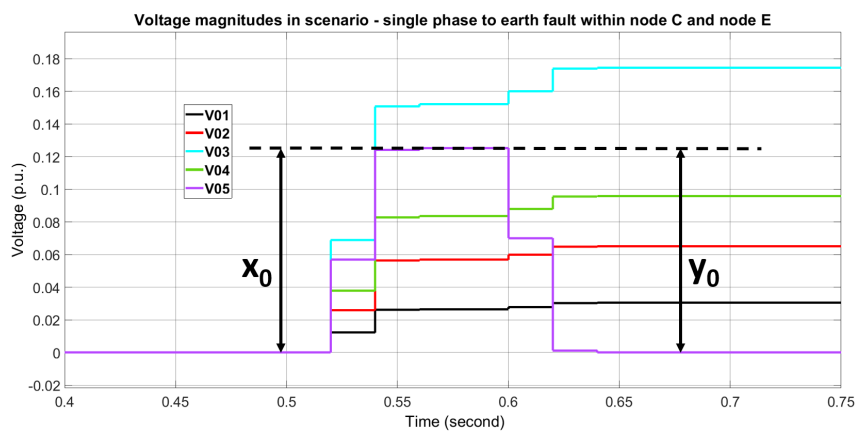

Figure 6: Zero sequence voltage magnitude of 5-bus system

acceptable, which have been proposed based on the results of extensive simulations and can be modified if necessary. Fig.6 shows the voltage behaviours for the fault scenario depicted in Fig.1. The variable $x_{0}$ indicates the maximum magnitude of the voltage rise and $y_{0}$ indicates any drop in voltages after the voltages have reached their maximum values. The fault is applied at $0.5 \mathrm{~s}$ in the simulation and it is assumed that $C B_{8}$ at node $\mathrm{C}$ operates correctly and opens $80 \mathrm{~ms}$ later at $0.58 \mathrm{~s}$. A positive value of the ratio of $y_{0}$ to $x_{0}$ is used to identify voltage recovery (which could be indicative of either partial or full recovery). A ratio of $y_{0}$ to $x_{0}$ of greater than 0.9 indicates full recovery - e.g. in $V_{05}$ in Fig.6. A negative value for $y_{0}$ indicates a further rise after the initial rise (e.g. $V_{01}, V_{02}, V_{03}$ and $V_{04}$ ).

It is very crucial to identify when a voltage has partially or fully recovered (indicating that a circuit breaker has opened and therefore protection has operated), and when it has gone up further due to the operation of a circuit breaker remote from the measured voltage location. These behaviour patterns provide indication for further analysis of circuit breaker status and faulted feeders. During and following fault events that are not properly responded to correctly, the only two scenarios that can be observed are similar to those of Three-phase fault scenarios. Scenario 1 is all elements in set $\boldsymbol{V}_{\text {oreg }}$ recover, but not all fully recover; while scenario 2 is only a subset of $\boldsymbol{V}_{\text {oreg }}$ have their voltage recovered. A new set of elements within set $\boldsymbol{N}_{\text {oreg }}$, from stage 1 containing all voltages that subsequently exhibit partial recovery or full recovery behaviour is denoted as set $\boldsymbol{N}_{\text {oup }}$. The remaining elements within the set $\boldsymbol{N}_{\text {oreg }}$, which have not partially recovered or fully recovered form the set $\boldsymbol{N}_{\text {oother }}$. In scenario 1 , if $V_{0 \max }$ has not recovered most significantly within set $\boldsymbol{V}_{\text {oreg }}$, then the faulted circuit is identified as the feeder connected between $N_{0 \max }$ and the node with the most significant voltage recovery within set $\boldsymbol{N}_{\text {oreg }}$. Operational failure of the protection/circuit breaker on this feeder at $N_{0 \max }$ can be reported. Otherwise, if $V_{0 \max }$ has recovered most significantly within the set $V_{0 r e g}$, then the faulted circuit is identified as the feeder connecting $N_{0 \max }$ and the node with the largest $k_{0 i}$ within set $\boldsymbol{N}_{\text {oreg }}$. Operational failure of the protection/circuit breaker on this feeder remote from $N_{0 \max }$ can be reported. In scenario 2 , if $V_{0 \max }$ has recovered, the faulted circuit is identified as the feeder connected between $N_{0 \max }$ and the node with the highest $k_{0 i}$ within $\boldsymbol{V}_{\text {oother }}$. Operational failure of the protection/circuit breaker at the node on this feeder remote from $N_{0 \max }$ can be reported. Otherwise, the faulted circuit is identified as the feeder within $N_{0 \max }$ and the node with the most significant voltage recovery within set $N_{\text {oup }}$. Operational failure of the protection/circuit breaker on this feeder at $N_{0 \max }$ can be reported.

For the scenario depicted in Fig.1, the resulting voltage profiles are presented in Fig.6. C is $N_{0 \max }$ and, at the initial stage, it can be deduced that the other node connected to the faulted feeder is one of either nodes B, D or E. From around $0.6 \mathrm{~s}$, there is no falling back behaviour for $V_{0 \max }\left(V_{03}\right)$, but $V_{05}$ has the most significant recovery (fully recovering to pre-fault from $0.64 \mathrm{~s})$. Accordingly, the faulted circuit is then identified as the feeder connected between $\mathrm{C}$ and $\mathrm{E}$. However, the protection or circuit breaker at $\mathrm{C}\left(\mathrm{CB}_{7}\right)$ has failed as the voltage remains ascent after the opening of the circuit breaker at $\mathrm{E}$ - note that all other voltages also remain ascent and do not fall back - in this case backup protection would be required to operate. The system would quickly report on the failure of the protection/circuit breaker at $\mathrm{C}$.

The operational methodology of phase-phase fault are the same as of unbalanced faults, apart from the quantity evaluated is negative sequence voltage magnitude. To enhance the focus of the paper, the details and repetitive procedure for phasephase fault operational methodology is not presented in this paper.

\section{Evaluation of Scheme Capability}

In order to determine the general applicability of the proposed scheme to a range of different network topologies and fault levels, it is important to quantify factors such as the highest detectable fault resistance and the magnitudes and thresholds of voltage dips that can be used to detect the presence of faults. A simplified system model is used to determine the highest detectable fault resistance and the associated minimum magnitudes of voltage depressions that would be observed at nodes for a fault with the aforementioned resistance value. This information could then be used to "set" the proposed backup protection scheme.

\subsection{Simplified equivalent system model}

In order to ascertain the highest detectable fault resistance for different scenarios and/or to determine the appropriate setting of voltage thresholds for fault identification, a 2-bus simplified equivalent model derived from the system under study has been developed, as shown in Fig. 7.

Since the structure of transmission and sub-transmission level networks is typically meshed, the equivalent model uses an "artificial" line $\left(Z_{\text {art }}\right)$ to represent the paths connected in parallel (in an interconnected system) with the line $Z_{L}$ that is directly connected between the nodes.

For any specific line within a network, the parameters of the equivalent 2-bus model can be calculated using data from fault level studies. Fig. 7 shows this, but for the reduced model, where the "artificial" line represents the impedance between the two nodes via the alternative parallel routes through the interconnected network. Fault scenarios 1 and 2 are solid single phase to earth faults at A and B respectively. Single phase to 


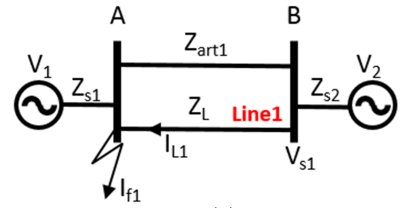

(a)

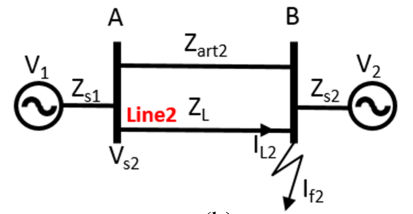

(b)
Figure 7: Single line diagram of simplified equivalent 2-bus model for system: (a) solid single phase to earth fault occurs at A. (b) solid single phase to earth fault occurs at B

earth faults are applied, since positive, negative and zero sequence components are all involved in a single phase to earth fault as shown in Fig. 8 (a) and (b), which are corresponding to scenarios of (a) and (b) in Fig. 7 respectively. As the parameters of the system will not change with variation of fault types, all positive, negative and zero sequence parameters of the system can be calculated in this situation.

For both (a) and (b) of Fig.8, from top to bottom, the circuits are orderly positive, negative and zero sequence. N0, N1, $\mathrm{N} 2$ are the neutral points of zero sequence, positive sequence and negative sequence circuits (the voltage magnitudes of neutral points are zero). The following are the parameters that don't change with variation of fault locations on line L: $V_{1}$ and $V_{2}$ are the source voltage without source impedances.

$Z_{s 1_{1} 1}$ and $Z_{s 1 \_2}$ are the positive sequence source impedances of G1 and G2 respectively. Similarly, $Z_{s 2_{-} 1}$ and $Z_{s 2_{2} 2}$ are the negative sequence source impedances and $Z_{s 0_{1} 1}$ and $Z_{s 0 \_2}$ are the zero sequence source impedances of $\mathrm{G} 1$ and $\mathrm{G} 2$ respectively.

$Z_{L 1}, Z_{L 2}$ and $Z_{L 0}$ are the positive sequence, negative sequence and zero sequence impedance of line $\mathrm{L}$.

$R_{f}$ is the fault resistance. As for both scenarios, solid fault is applied, $R_{f}$ is 0 when calculating equivalent circuit parameters. For the scenario that fault happens at node A (as shown in (a) of Fig.8) - scenario 1: $V_{1_{-} 1}$ and $V_{2 \_}$are the calculated internal source voltages without impact of source impedance.

$V_{A 1_{1} 1}$ and $V_{B 1_{-} 1}$ are the positive sequence source voltages that can be directly measured from A and B. Similarly, $V_{A 2_{-} 1}$ and $V_{B 2 \_1}$ are the negative sequence source voltage and $V_{A 0_{-} 1}$ and $V_{B 0 \_1}$ are the zero sequence source voltage of node A and B respectively.

$Z_{\text {art } 1_{\_} 1}, Z_{\text {art } 2_{-} 1}$ and $Z_{\text {art } 0_{-} 1}$ are the positive sequence, negative sequence and zero sequence impedance of the "artificial line" which is an equivalent of the interconnection between node A and $\mathrm{B}$.

$V_{L 1 \_1}, V_{L 2 \_1}$ and $V_{L 0_{-} 1}$ are the positive sequence, negative sequence and zero sequence voltage drop of line L / the "artificial line".

$I f_{1 \_1}, I f_{2_{-} 1}$ and $I f_{0_{-} 1}$ are the positive sequence, negative sequence and zero sequence total fault current at the fault location (node $\mathrm{A}$ in this scenario).

$I_{L 1 \_1}, I_{L 2 \_1}$ and $I_{L 0 \_1}$ are the positive sequence, negative sequence and zero sequence current flowing through line L towards fault.

$I_{2 f_{-} 1}$ is the positive sequence current flow from G2 towards fault.

For the scenario that fault happens at node B - scenario 2, the quantities marked in (b) of Fig.8 have the similar meanings to the quantities marked in (a) of Fig.8 as explained above. All the parameters in the system (both measured quantity and calculated quantity) are phasors, which include both magnitudes and angles.

As the sequence component circuits are connected in series when single-phase to earth fault is applied (for both scenarios), total fault current for sequence components are the same $\left(I_{f 1 \_1}\right.$ $\left.=I_{f 2_{-} 1}=I_{f 0_{-} 1} ; I_{f 1 \_2}=I_{f 2 \_2}=I_{f 0_{-} 2}\right)$.

In order to calculate the parameters of the 2-bus equivalent model, the following parameters are needed to be measured/calculated in the full model:

1. Fault current flowing through line $\mathrm{L}$ towards the fault: $I_{L 1 \_1}, I_{L 2 \_1}$ and $I_{L 0_{-} 1}$ for scenario 1 and $I_{L 1 \_2}, I_{L 2 \_2}$ and $I_{L 0 \_2}$ for scenario 2 .

2. Voltage magnitudes at node A and node B (source magnitudes with consideration of source impedance): $V_{A 1 \_1}$, $V_{B 1 \_2}, V_{A 2_{-} 1}, V_{B 2_{-} 1}, V_{A 0_{-} 1}$ and $V_{B 0_{-} 1}$ for scenario 1. $V_{A 1 \_2}$, $V_{B 1 \_2}, V_{A 2 \_2}, V_{B 2 \_2}, V_{A 0 \_2}$ and $V_{B 2 \_2}$ for scenario 2 .

3. Total fault current at fault location: $I_{f 1_{-} 1}, I_{f 2_{-} 1}$ and $I_{f 0_{-} 1}$ for scenario 1 and $I_{f 1 \_2}, I_{f 2 \_}$and $I_{f 0 \_2}$ for scenario 2.

With the values measured/ calculated above, the followings are the target parameters needs to be identified for 2-bus equivalent model:

1. Voltage source internal impedance: $Z_{s 1_{-} 1}, Z_{s 1_{-} 2}, Z_{s 2_{-} 1}$, $Z_{s 2 \_2}, Z_{s 0 \_1}$ and $Z_{s 0 \_2}$.

2. Impedance of the "artificial line" $Z_{\text {art } 1_{-} 1}, Z_{\text {art2_1 }}, Z_{\text {art } 1 \_2}$, $Z_{\text {art } 2 \_2}, Z_{\text {art } 0 \_1}$, and $Z_{\text {art } 0 \_2}$.

3. Source voltage magnitudes without internal source impedance: $V_{1}$ and $V_{2}$.

All the parameters in the system (both measured quantity and calculated quantity) are phasors, which include both magnitudes and angles.

\subsubsection{Negative sequence parameter calculation}

To calculate negative sequence parameters of the 2-bus equivalent circuit, Equation (1) is created based on Kirchhoff's Voltage Law (KVL); Equation (2) is created based on Kirchhoff's Current Law and (KCL) and Equation (3) is based on KCL and Ohm's law. Equation (1) is to represent intermediate quantity $V_{L 2 \_1}$ and $V_{L 2 \_2}$ by other quantities (either are the known ones or the ones remain to be solved). $Z_{s 2_{2} 1}$ and $Z_{s 2 \_2}$ can be calculated by Equation (1) and Equation (2). $Z_{\text {art2_1 }}$ and $Z_{\text {art } 2 \_2}$ can be calculated by equation Equation (1) and Equation (3).

$$
\begin{aligned}
& \left\{\begin{array}{l}
V_{L 2 \_1}=I_{L 2 \_1} \times Z_{L 2} \\
V_{L 2 \_2}=I_{L 2 \_2} \times Z_{L 2}
\end{array}\right. \\
& \left\{\begin{array}{l}
-I_{f 2_{-} 1}=V_{A 2_{-} 1} / Z_{s 2_{-} 1}+\left(V_{A 2 \_1}+V_{L 2 \_1}\right) / Z_{s 2_{-} 2} \\
-I_{f 2_{-} 2}=V_{B 2_{-} 2} / Z_{s 2_{2} 2}+\left(V_{B 2 \_2}+V_{L 2 \_1}\right) / Z_{s 2_{-} 1}
\end{array}\right. \\
& \left\{\begin{array}{l}
Z_{a r t 2 \_1}=V_{L 2 \_1} /\left(-\left(V_{A 2 \_1}+V_{L 2_{1}}\right) / Z_{s 2 \_2}-I_{L 2 \_1}\right) \\
Z_{a r t 2 \_2}=V_{L 2 \_2} /\left(-\left(V_{B 2 \_2}+V_{L 2_{2}}\right) / Z_{s 2 \_1}-I_{L 2 \_2}\right)
\end{array}\right.
\end{aligned}
$$






(a)

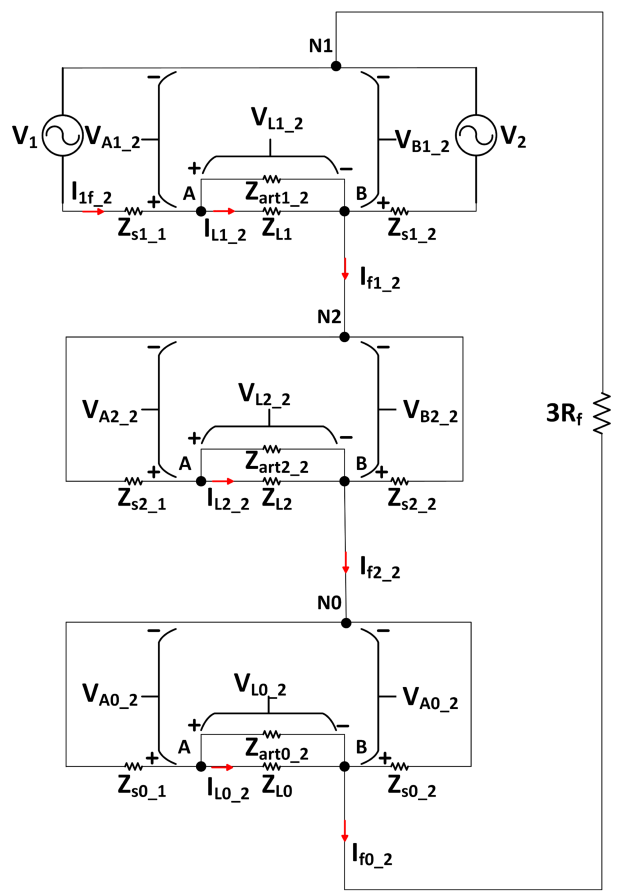

(b)

Figure 8: Sequence components of single-phase to earth fault: (a) fault at A (b) fault at B

\subsubsection{Zero sequence network parameter calculation}

Similarly with negative sequence, in order to calculate zero sequence parameters of the 2-bus equivalent circuit, Equation (4) is created based on KVL; Equation (5) is created based on KCL and Equation (6) is based on KCL and Ohm's law. $Z_{s 0_{1} 1}$ and $Z_{S 0 \_2}$ can be calculated by Equation (4) and Equation (5). $Z_{\text {art_1 }}$ and $Z_{\text {art } 0_{-2}}$ can be calculated by equation Equation (4) and Equation (6).

$$
\begin{aligned}
& \left\{\begin{array}{l}
V_{L 0 \_1}=I_{L 0 \_1} \times Z_{L 0} \\
V_{L 0 \_2}=I_{L 0 \_2} \times Z_{L 0}
\end{array}\right. \\
& \left\{\begin{array}{l}
-I_{f 0_{-} 1}=V_{A 0_{-} 1} / Z_{s 0_{-} 1}+\left(V_{A 0 \_1}+V_{L 0_{-} 1}\right) / Z_{s 0_{-} 2} \\
-I_{f 0 \_2}=V_{B 0_{-} 2} / Z_{s 0_{-} 2}+\left(V_{B 0_{-} 2}+V_{L 0_{-} 1}\right) / Z_{s 0 \_1}
\end{array}\right. \\
& \left\{\begin{array}{l}
Z_{a r t 0 \_1}=V_{L 0 \_1} /\left(-\left(V_{A 0 \_1}+V_{L 0 \_1}\right) / Z_{S 0 \_2}-I_{L 0 \_1}\right) \\
Z_{a r t 0 \_2}=V_{L 0 \_2} /\left(-\left(V_{B 0 \_2}+V_{L 0 \_2}\right) / Z_{S 0 \_1}-I_{L 0 \_2}\right)
\end{array}\right.
\end{aligned}
$$

As the assumed equivalent circuit ignores shunt elements such as line capacitances, the calculated impedance of the "artificial line" based on scenario 1 and scenario $2\left(Z_{\text {art2_1 }}\right.$ and $Z_{\text {art2_2 }}$ for negative sequence; $Z_{a r t 0_{1} 1}$ and $Z_{a r t 0_{2}}$ for zero sequence) may have slightly different values compared to the actual situation based on a full network model. However, it has been determined that the difference in practical situations is not significant (less than $0.18 \%$ for IEEE 14-bus system), and therefore, will not impact on the overall capability and performance of the scheme.

For the validation of the scheme, $Z_{\text {art } 2}$ (negative sequence impedance of the "artificial line") and $Z_{\text {art } 0}$ (zero sequence impedance of the "artificial line") are therefore taken as an average of the value calculated in scenario 1 and scenario 2 to minimize the error introduced by any limitations of the assumed equivalent circuit, as demonstrated in Equation (7).

$$
\left\{\begin{array}{l}
Z_{a r t 2}=\left(Z_{\text {art } 2 \_1}+Z_{\text {art } 2 \_2}\right) / 2 \\
Z_{\text {art } 0}=\left(Z_{\text {art } 0 \_1}+Z_{\text {art } 0 \_2}\right) / 2
\end{array}\right.
$$

\subsubsection{Positive sequence parameters calculation}

For positive sequence, line impedances and source impedances are the same as the negative sequence, which are presented in Equation (8).

$$
\left\{\begin{array}{l}
Z_{s 1 \_1}=Z_{s 2 \_1} \\
Z_{s 1 \_2}=Z_{s 2 \_2} \\
Z_{a r t 1}=Z_{\text {art } 2} \\
Z_{L 1}=Z_{L 2}
\end{array}\right.
$$

Internal source voltage (without source impedance) can be calculated in positive sequence circuit. Similarly to the calculation of impedance of "artificial line", the neglect of shunt elements such as line capacitances in equivalent circuit, the calculated internal source voltages without source impedance-based on scenario 1 and scenario $2\left(V_{1 \_1}\right.$ and $V_{2 \_1}$ under scenario 1 and $V_{1 \_2}$ and $V_{2 \_2}$ under scenario 2) may have slightly different values compared to the actual situation based on a full network model. However, it has been determined that the difference in practical situations does not have significant impact to the scheme (less than $0.18 \%$ difference in calculated 'artificial line' impedance for IEEE 14-bus system), and therefore, will not have impact on the overall capability and performance of 
the scheme. To minimize the error introduced by any limitations of the assumed equivalent circuit, $V_{1}$ and $V_{2}$ are taken as an average of the value calculated in scenario 1 and scenario 2, as shown in Equation (13).

For scenario 1, as shown in Equation (9), intermediate quantity $I_{2 f_{-} 1}$ is represented by known quantity $I_{L 1_{-} 1}$ and calculated quantities $Z_{\text {art } 1}$ and $Z_{L 1}$ based on Kirchhoff's Current Law (KCL).

$$
I_{2 f_{-} 1}=I_{L 1_{-} 1} / Z_{\text {art } 1} \times\left(Z_{L 1}+Z_{\text {art } 1}\right)
$$

Internal source voltages without source impedance $\left(V_{1_{-} 1}\right.$ and $V_{2 \_}$) can be calculated by Equation (10), which is created based on Kirchhoff's Voltage Law (KVL) and Ohm's law.

$$
\left\{\begin{array}{l}
V_{1 \_1}=V_{A 1 \_1}+I_{2 f_{-1}} \times Z_{s 1_{\_} 2}+I_{L 1 \_1} \times Z_{L 1} \\
V_{2 \_1}=V_{A 1 \_1}+Z_{s 1_{-} 1} \times\left(I_{f 1 \_1}-I_{2 f_{-1}}\right)
\end{array}\right.
$$

For scenario 2, as shown in Equation (11), intermediate quantity $I_{1 f_{-} 2}$ is represented by known quantity $I_{L 1 \_2}$ and calculated quantities $Z_{a r t 1}$ and $Z_{L 1}$ based on Kirchhoff's Current Law (KCL).

$$
I_{1 f_{-} 2}=I_{L 1 \_2} / Z_{\text {art } 1} \times\left(Z_{L 1}+Z_{\text {art } 1}\right)
$$

Internal source voltages without source impedance $\left(V_{1_{-} 1}\right.$ and $V_{2 \_}$) can be calculated by Equation (12), which is created based on Kirchhoff's Voltage Law (KVL) and Ohm's law.

$$
\begin{gathered}
\left\{\begin{array}{c}
V_{1 \_2}=V_{B 1 \_2}+I_{1 f \_2} \times Z_{s 1 \_1}+I_{L 1 \_2} \times Z_{L 1} \\
V_{2 \_2}=V_{B 1 \_2}+Z_{s 1 \_2} \times\left(I_{f 1 \_2}-I_{1 f \_2}\right)
\end{array}\right. \\
\left\{\begin{array}{l}
V_{1}=\left(V_{1 \_1}+V_{1 \_2}\right) / 2 \\
V_{2}=\left(V_{1 \_2}+V_{2 \_}\right) / 2
\end{array}\right.
\end{gathered}
$$

\subsection{Configuration of the developed scheme}

The worst case with respect to fault detection for a threephase fault is when a fault is at a location on the feeder which results in the lowest positive sequence terminal voltage depression for a fixed fault resistance.The worst-case with respect to fault detection for an unbalanced earth fault or phase-phase fault is when a fault is at a location on the feeder which results in the lowest zero (unbalanced earth fault) or negative (phasephase fault) sequence terminal voltage increase for a fixed fault resistance. In order to find the highest detectable fault resistance for a pre-determined voltage threshold, the worst-case fault position on the line needs to be identified, which results in the minimum positive sequence voltage depression (for threephase faults) or minimum zero (unbalanced earth fault) or negative (phase-phase fault) voltage increase at the line end. If a fault with a resistance that can be detected in the aforementioned worst-case scenario, then it is considered that this resistive fault is also delectable in all the other locations in the line.

For the proposed scheme, it considers there is a fault if the voltage of at least one of the two nodes connected to the faulted line is less than a threshold. In order to find the highest detectable resistive fault for a given network, a method for automatically identifying this worst case and highest detectable

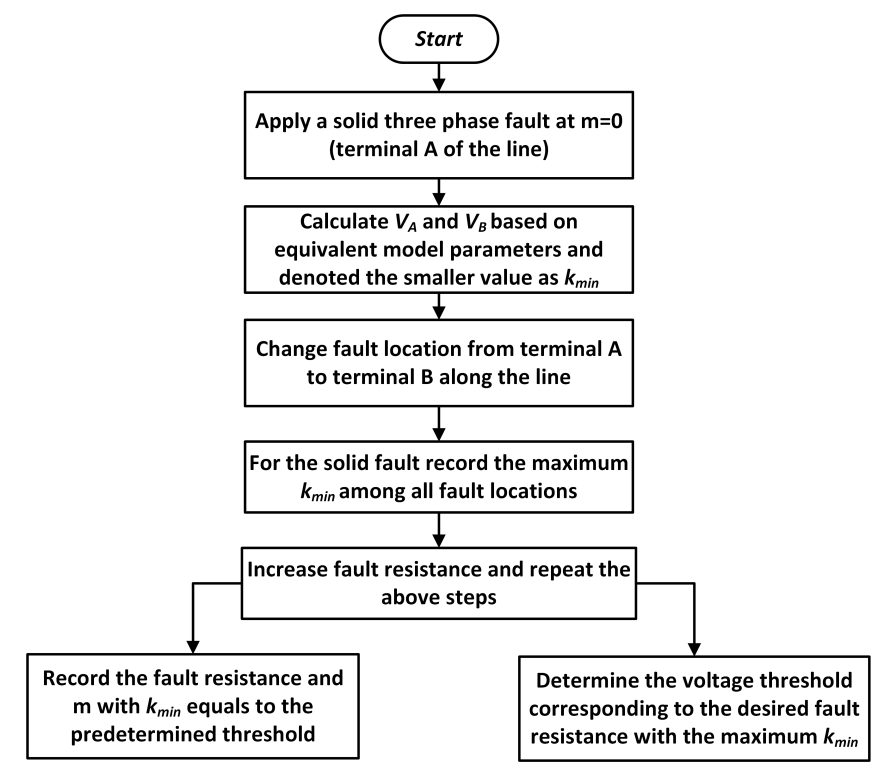

Figure 9: Flow chart of configuration determination

fault resistance, has been developed. This information can be used to specify the voltage thresholds for a given maximum fault resistance, or conversely to specify the maximum fault resistance that could be detected for a pre-determined voltage threshold.

Four steps are included in the method. As the calculations are based on the simple 2-bus equivalent circuit and the the parameters have already been calculated in Section 3.1, the overall process is much simpler and faster compared to a simulationbased solution using a full power network model. For a three phase fault, the steps are demonstrated in Figure 9 and explained below.

1. Step 1: For a fixed feeder with solid fault with $\mathrm{m}=0$ (fault location on the feeder at node A), calculate $V_{A}$ and $V_{B}$ and choose the smaller value of $V_{A}$ and $V_{B}$, denoted as $k_{\text {min }}$. Record $k_{\text {min }}$.

2. Step 2: Increase the value of $m$ from 0 to 1 incrementally (fault location on the feeder from node A to node B) and repeat step 1 . Find the maximum $k_{\min }$ and corresponding value of $m$ and record these as the worst-case values.

3. Step 3: Repeat step 2 with fault resistance increasing from 0 to a suitably high fault resistance value.

4. Step 4: If the target is to find the highest detectable fault resistance for a predetermined voltage threshold, then the value of fault resistance corresponding to the scenario when $k_{\text {min }}$ is equal to the predetermined voltage threshold is determined, based on the results of step 3. This is shown for the IEEE 14-bus system in Fig. 10. Similarly, voltage thresholds can also be determined for a given maximum fault resistance.

Sharing the same principle, for an unbalanced earth fault (or phase-phase fault), the steps are explained as below.

1. Step 1: For a fixed feeder with solid single phase to earth fault with $\mathrm{m}=0$ (fault location on the feeder at node A), calculate $V_{A 01}$ and $V_{B 01}\left(V_{A 01}\right.$ and $V_{B 01}$ for phasephase fault) and choose the larger value of $k_{0 A 1}$ and $k_{0 B 1}$ 
( $k_{0 A 1}$ and $k_{0 B 1}$ represent for the difference between "during fault" zero sequence voltage magnitude and pre-fault zero sequence voltage magnitude of node $\mathrm{A}$ and $\mathrm{B}$ respectively), denoted as $k_{0 \max }$. Record $k_{0 \max }$.

2. Step 2: Increase the value of $m$ from 0 to 1 incrementally (fault location on the feeder from node A to node B) and repeat step 1 . Find the minimum value of $k_{0 \max }$ and corresponding value of $\mathrm{m}$ and record these as the worst-case values.

3. Step 3: Repeat step 2 with fault resistance increasing from 0 to a suitably high fault resistance value.

4. Step 4: If the target is to find the highest detectable fault resistance for a predetermined voltage threshold, then the value of fault resistance corresponding to the scenario when $k_{0 \text { max }}$ is equal to the predetermined voltage threshold is determined, based on the results of step 3. Similarly, voltage thresholds can also be determined for a given maximum fault resistance.

\section{Case Studies}

The operation of the scheme presented in Section 2 has been demonstrated using case studies conducted by Simscape Electrical package based upon the IEEE 14-bus system as shown in Fig. 10. The studies only consider the $132 \mathrm{kV}$ system elements. Measurements are collected from PMU models created by the University of Strathclyde $(10 \mathrm{kHz}, \mathrm{P}$ class with a reporting rate of $50 \mathrm{~Hz}$ )[25-27].

\subsection{Simplified equivalent system model}

The 14-bus model is used to derive a set of 2-bus simplified models (as described in Section 3.1). The calculated 2-bus equivalent circuit parameters for each of the $132 \mathrm{kV}$ lines ( 7 in total) in the 14-bus model are as shown in Table 1.

Slight differences exist between $Z_{\text {art 1_1 }}$ and $Z_{\text {art 1_2 }}$ (between $Z_{\text {art } 0 \_1}$ and $Z_{\text {art } 0 \_2}$ ), which can be quantified using Equation (14). As can be seen from Table 1 the difference is less than $0.18 \%$ for all lines which is considered acceptable and can be neglected. $Z_{\text {art } 1}$ (and $Z_{\text {art } 0}$ ) is then defined as an average value (complex number)of $Z_{\text {art } 1 \_1}$ and $Z_{\text {art } 1 \_2}\left(Z_{\text {art } 0_{-} 1}\right.$ and $\left.Z_{\text {art } 0 \_2}\right)$ to minimize any error introduced by this equivalent circuit approach.

$$
\left\{\begin{array}{l}
\Delta Z_{a r t 1}=\left|Z_{\text {art } 1 \_1}-Z_{\text {art } 1 \_2}\right| / \min \left(Z_{\text {art } 1 \_1}, Z_{\text {art } 1 \_2}\right) \\
\Delta Z_{\text {art } 0}=\left|Z_{\text {art } 0 \_1}-Z_{\text {art } 0 \_2}\right| / \min \left(Z_{\text {art } 0 \_1}, Z_{\text {art } 0 \_2}\right)
\end{array}\right.
$$

\subsection{Highest detectable fault resistance identification and threshold setting calculation}

According to the 2-bus equivalent parameters presented in Table 1 and the method explained in Section 3.2, the highest detectable resistance corresponding to predetermined voltage threshold settings can be established.

For a specific feeder, the first step is to define a voltage threshold that can be used to identify the presence of faults.

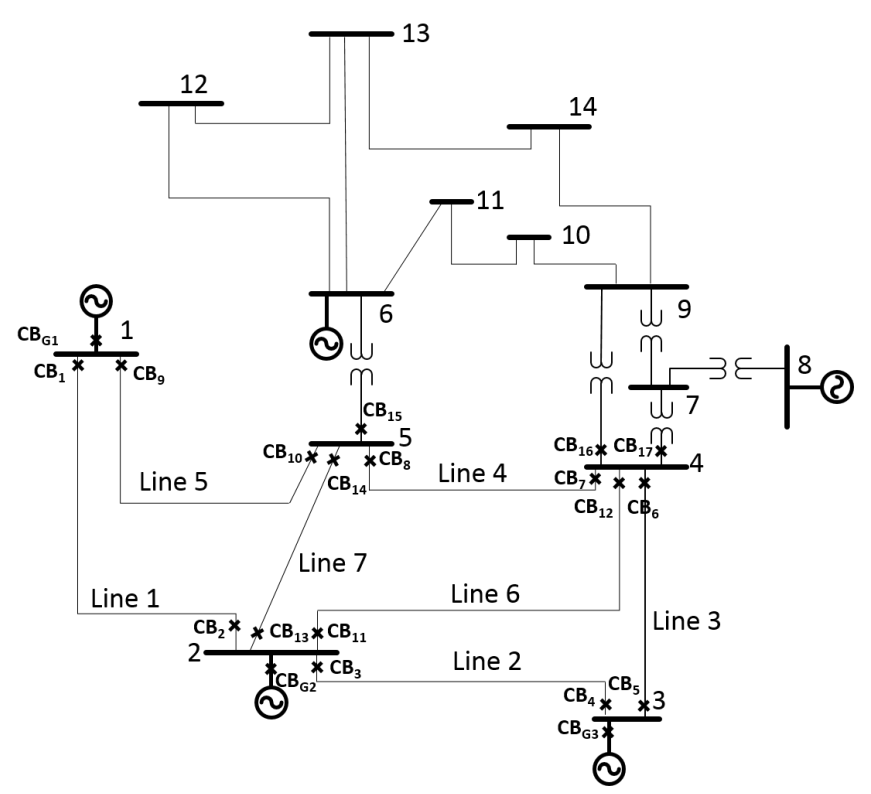

Figure 10: Single line diagram of IEEE 14-bus system

The second step is to calculate the "worst case" for all scenarios with resistance from 0 to a relatively high resistance. The final step is to identify the highest detectable resistance with results from the second step as demonstrated in Section 3.2.

In order to test and validate the method, a threshold of $85 \%$ of pre-fault voltage is specified for three phase faults, which is step one. As the threshold is a proportional value of pre-fault voltage, variations in the prevailing pre-disturbance voltage will not impact the scheme. For the second step, all worst-case scenarios for fault resistance from 0 to a relatively high resistance are automatically calculated as reference for the final step. For illustrative purposes, the results for line 3 are shown in Fig. 11. It can be observed that with a voltage depression threshold of $15 \%$, the fault resistances for scenarios when terminal voltage $\left(k_{1}\right)$ is just at the threshold are between 30 and $40 \Omega$. The fault resistance calculated in scenario when $V_{B}$ is equal to the threshold is identified as the highest detectable fault resistance for line 3 (with $m=0.62$ as shown in Fig. 11 (b)) - the final step. Using this threshold, the maximum fault resistances that will be detected as faults are shown in Table 2 . The values for $\mathrm{m}$ indicate the fault location for the highest detectable fault resistance which results in the smallest voltage depression. For example, for line $3, R_{f \max }$ is selected for the scenario where $V_{B}$ (node 4 in Fig. 10) is $85 \%$ of the initial voltage. The worst case corresponds to $\mathrm{m}=0.62$ (i.e. a fault at a location $62 \%$ of the line length away from node 3 in Fig. 10).

Table 2 indicates that the proposed scheme can be used to analyse the protection performance anywhere within the test system for fault resistances up to $34.6 \Omega$, although using higher voltage thresholds would increase the maximum detectable fault resistance, at the potential expense of triggering system operation for non-fault transients - the "strength" or prevailing fault levels in the system could be used to configure the voltage thresholds and corresponding maximum detectable fault resistances in an actual application. 


\begin{tabular}{|c|c|c|c|c|c|c|}
\hline \multicolumn{7}{|c|}{ Positive (and negative) sequence $(\Omega)$} \\
\hline Line & $Z_{s 1 \_1}(\Omega)$ & $Z_{s 1 \_2}(\Omega)$ & $Z_{a r t 11_{1}}(\Omega)$ & $Z_{\text {art } 122}(\Omega)$ & $Z_{\text {art } 1}(\Omega)$ & $\Delta Z_{\text {art } 1}$ \\
\hline 1 & $11.01 \angle 1.47$ & $28.38 \angle 1.44$ & $70.21 \angle 1.27$ & $70.33 \angle 1.27$ & $70.27 \angle 1.27$ & $0.18 \%$ \\
\hline 2 & $14.21 \angle 1.40$ & $48.75 \angle 1.44$ & $60.47 \angle 1.17$ & $60.46 \angle 1.17$ & $60.47 \angle 1.17$ & $0.02 \%$ \\
\hline 3 & $39.24 \angle 1.45$ & $21.38 \angle 1.38$ & $86.04 \angle 1.25$ & $86.01 \angle 1.26$ & $86.03 \angle 1.25$ & $0.03 \%$ \\
\hline 4 & $34.08 \angle 1.37$ & $23.68 \angle 1.41$ & $32.45 \angle 1.16$ & $32.44 \angle 1.16$ & $32.44 \angle 1.16$ & 0 \\
\hline 5 & $15.69 \angle 1.41$ & $34.58 \angle 1.43$ & $24.13 \angle 1.23$ & $24.12 \angle 1.23$ & $24.13 \angle 1.23$ & $0.03 \%$ \\
\hline 6 & $10.29 \angle 1.47$ & $32.50 \angle 1.44$ & $30.56 \angle 1.22$ & $30.56 \angle 1.22$ & $30.56 \angle 1.22$ & 0 \\
\hline 7 & $16.31 \angle 1.41$ & $29.87 \angle 1.45$ & $23.38 \angle 1.24$ & $23.37 \angle 1.24$ & $23.38 \angle 1.24$ & $0.13 \%$ \\
\hline \multicolumn{7}{|c|}{ Zero sequence $(\Omega)$} \\
\hline Line & $Z_{s 0 \_1}(\Omega)$ & $Z_{s 0 \_2}(\Omega)$ & $Z_{\text {art0_1 }}(\Omega)$ & $Z_{\text {art0_2 }}(\Omega)$ & $Z_{\text {art } 0}(\Omega)$ & $\Delta Z_{a r t 0}$ \\
\hline 1 & $6.95 \angle 1.47$ & $30.44 \angle 1.44$ & $182.56 \angle 1.30$ & $182.66 \angle 1.31$ & $182.61 \angle 1.30$ & $0.06 \%$ \\
\hline 2 & $18.80 \angle 1.38$ & $39.56 \angle 1.46$ & $161.12 \angle 1.20$ & $166.11 \angle 1.19$ & $166.11 \angle 1.19$ & $0 \%$ \\
\hline 3 & $33.24 \angle 1.45$ & $57.41 \angle 1.31$ & $398.91 \angle 1.24$ & $398.60 \angle 1.24$ & $398.75 \angle 1.24$ & $0.08 \%$ \\
\hline 4 & $66.28 \angle 1.29$ & $64.25 \angle 1.33$ & $465.02 \angle 1.14$ & $465.09 \angle 1.14$ & $465.06 \angle 1.14$ & $0.02 \%$ \\
\hline 5 & $17.91 \angle 1.39$ & $92.66 \angle 1.33$ & $87.03 \angle 1.22$ & $86.99 \angle 1.22$ & $87.01 \angle 1.22$ & $0.05 \%$ \\
\hline 6 & $6.47 \angle 1.46$ & $85.50 \angle 1.35$ & $122.48 \angle 1.18$ & $122.34 \angle 1.17$ & $122.41 \angle 1.18$ & $0.11 \%$ \\
\hline 7 & $17.95 \angle 1.38$ & $90.08 \angle 1.35$ & $89.81 \angle 1.23$ & $89.78 \angle 1.23$ & $89.79 \angle 1.23$ & $0.04 \%$ \\
\hline
\end{tabular}

Table 1: Calculated parameters for 2-bus equivalent circuits

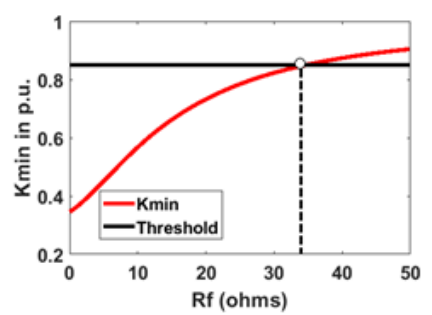

(a)

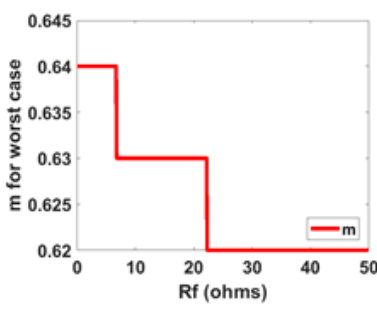

(b)
Figure 11: Highest detectable fault resistance identification for line 3: (a) relationship between $k_{3}$ and $R_{f}$ (b) relationship between worst case fault locations and $R_{f}$

\begin{tabular}{c|c|c|c|c}
\hline Line & $R_{f \max }(\Omega)$ & $\mathbf{m}$ & Threshold voltage & Case \\
\hline 1 & 16.6 & 0 & $k_{A}$ & 1 \\
\hline 2 & 25 & 0 & $k_{A}$ & 2 \\
\hline 3 & 34.7 & 0.62 & $k_{B}$ & 3 \\
\hline 4 & 31.7 & 0.62 & $k_{B}$ & 4 \\
\hline 5 & 16.6 & 0 & $k_{A}$ & 5 \\
\hline 6 & 25 & 0 & $k_{A}$ & 6 \\
\hline 7 & 25 & 0 & $k_{A}$ & 7 \\
\hline
\end{tabular}

Table 2: Highest fault resistance for different feeders

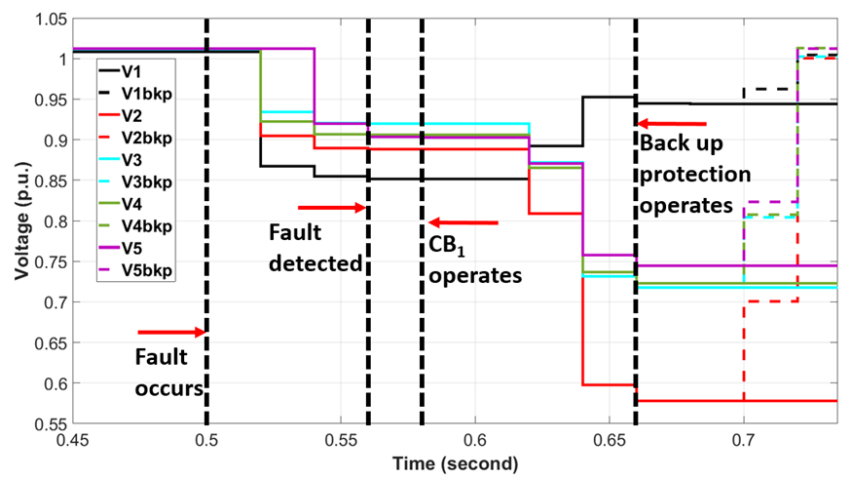

Figure 12: Test results for fault on Line 1

\subsection{Case study results and validation of the proposed scheme}

Fig. 12 demonstrates operation of the scheme via a "timeline" illustrating the positive sequence voltage magnitude profiles for a scenario where Line 1 is faulted with a three phase fault. Solid lines and dashed voltage traces represent the system without and with the backup scheme respectively. The detection of the fault and the backup protection operation occur at $0.56 \mathrm{~s}$ and $0.66 \mathrm{~s}$ respectively.

In order to validate the operation of the scheme, faults with a corresponding protection/circuit breaker failure at one line end for all $132 \mathrm{kV}$ lines in the system (under the worst-case scenarios) have been simulated and the scheme responses are shown in Table 3, where FR represents faulted region and FF represents faulted feeder. Case 1-7 correspond to the scenarios shown in Table 2. $C B$ is the protection/breaker that operates correctly, while $C B_{f}$ is the failed protection/breaker. This means that there are two case studies for each line corresponding to a failure of protection/breaker at each line end. $t_{f o}$ is 


\begin{tabular}{c|c|c|c|c|c|c|c}
\hline \multicolumn{2}{c|}{ Scenarios } & \multicolumn{6}{c}{ Outputs } \\
\hline Case & $\mathrm{CB}$ & $\mathrm{FR}$ & $t_{f o}(\mathrm{~s})$ & $\mathrm{FF}$ & $t_{f l}(\mathrm{~s})$ & $C B_{f}$ & $C B_{b c k}$ \\
\hline \multirow{2}{*}{1} & 1 & 1,5 & 0.6 & 1 & 0.66 & 2 & $3,11,13, \mathrm{G} 2$ \\
\cline { 2 - 8 } & 2 & 1,5 & 0.6 & 1 & 0.66 & 1 & $9, \mathrm{G} 1$ \\
\hline \multirow{2}{*}{2} & 3 & $1,2,6,7$ & 0.6 & 2 & 0.66 & 4 & $5, \mathrm{G} 3$ \\
\cline { 2 - 8 } & 4 & $1,2,6,7$ & 0.6 & 2 & 0.66 & 3 & $2,11,13, \mathrm{G} 2$ \\
\hline \multirow{2}{*}{3} & 5 & $1,2,6,7$ & 0.6 & 3 & 0.66 & 6 & $7,12,16,17$ \\
\cline { 2 - 8 } & 6 & $1,2,6,7$ & 0.6 & 3 & 0.66 & 5 & $4, \mathrm{G} 3$ \\
\hline \multirow{3}{*}{4} & 7 & $4,5,7$ & 0.6 & 4 & 0.66 & 8 & $10,14,15$ \\
\cline { 2 - 8 } & 8 & $4,5,7$ & 0.6 & 4 & 0.66 & 7 & $6,12,16,17$ \\
\hline \multirow{2}{*}{5} & 9 & $4,5,7$ & 0.6 & 5 & 0.66 & 10 & $10,14,15$ \\
\cline { 2 - 8 } & 10 & $4,5,7$ & 0.6 & 5 & 0.66 & 9 & $1, \mathrm{G} 1$ \\
\hline \multirow{2}{*}{6} & 11 & $1,2,6,7$ & 0.6 & 6 & 0.68 & 12 & $6,7,16,17$ \\
\cline { 2 - 8 } & 12 & $1,2,6,7$ & 0.6 & 6 & 0.68 & 11 & $2,3,13, \mathrm{G} 2$ \\
\hline \multirow{2}{*}{7} & 13 & $1,2,6,7$ & 0.6 & 7 & 0.68 & 14 & $8,10,15$ \\
\cline { 2 - 8 } & 14 & $1,2,6,7$ & 0.6 & 7 & 0.68 & 13 & $2,3,11$ \\
\hline
\end{tabular}

Table 3: Test results of three phase faults

the time of fault identification (all faults occur at $0.5 \mathrm{~s}$ with the operational circuit breaker opening at $0.58 \mathrm{~s}$ ). $C B_{b c k}$ represents the protection/circuit breakers which are subsequently sent tripping signals to effect backup protection. All faults applied are three-phase resistive faults. $t_{f l}$ is the time when the analysis is complete and the system has reported upon the faulted circuit and failed protection/breaker.

It is clear from the test results that the scheme operates correctly and in a timely fashion for all of the 14 tests under "worst case" fault resistance scenarios. For all cases, fault occurrence and the faulted region are identified within $80 \mathrm{~ms}$ after fault occurrence and the precise fault location (in terms of the faulted circuit) and failed protection/circuit breaker can be identified within a further $100 \mathrm{~ms}$ after operation of one of the line-end circuit breakers. Thus, the overall operation time of the system before sending tripping signals is always less than $180 \mathrm{~ms}$ in this case. There may be small additional delays associated with PMU data concentration and communications, but these delays are not expected to be sufficiently lengthy to compromise the applicability of the scheme, which will be discussed in detail in Section 4.4.

With similar test procedure, Fig. 13 demonstrates the operation of the scheme with zero sequence profiles for a scenario where Line 1 is faulted with a single phase to earth fault. Solid lines and dashed voltage traces represent the system without and with the backup scheme respectively. Fault occurs at $0.2 \mathrm{~s}$ with $C B 1$ operates at $0.28 \mathrm{~s}$. The detection of the fault and the backup protection operation occur at $0.26 \mathrm{~s}$ and $0.36 \mathrm{~s}$ respectively.

With a $2 \%$ of zero sequence threshold, the tested results are presented in Table 4 . The threshold $2 \%$ is a reasonable threshold, since the typical accuracy classes for voltage transformers are $0.3 \%, 0.6 \%$ and $1.2 \%$ for voltages within range of $\pm 10 \%$ of nominal range [28]. Based on sequence-component theory, under the boundary scenario (high impedance fault), where zero

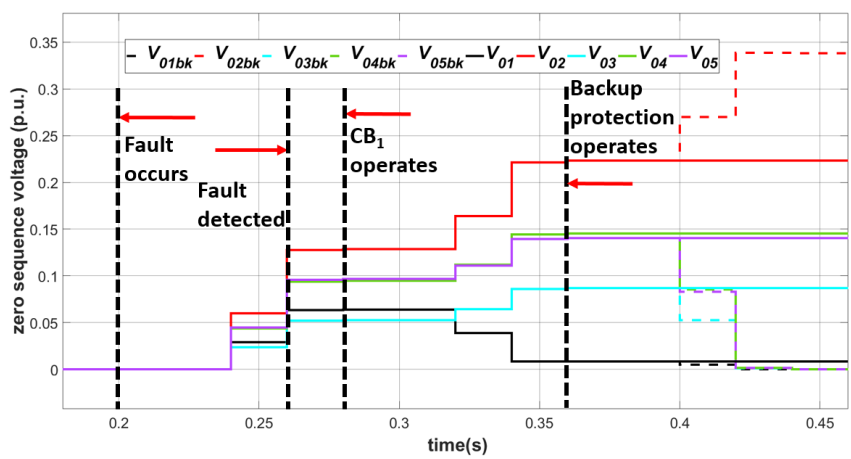

Figure 13: Test results for singe phase to earth fault on Line 1

sequence voltage equals to $2 \%$ of the initial voltage magnitude, the phase magnitude of the faulted phase is $94 \%$ of the nominal voltage, which is within the range of $\pm 10 \%$ of nominal range and therefore accuracy classes are $0.3 \%, 0.6 \%$ and $1.2 \%$. Since the phase voltage drop in this scenario is $6 \%$ which is much higher than the maximum acceptable errors of transformer [29]. Therefore the $2 \%$ threshold is reasonable with consideration of accuracy of voltage transformers.

For all cases, fault resistance varies from $1 \Omega$ to $241 \Omega$ with increment of $20 \Omega$ for each line and the highest detectable fault resistance for single phase to earth faults from the tests can be up to $241 \Omega$. Fault occurrence and the faulted region identification are within $100 \mathrm{~ms}$ after fault occurrence and the precise fault location (in terms of the faulted circuit) and failed protection/circuit breaker identification are within a further $100 \mathrm{~ms}$ after operation of one of the line-end circuit breakers. Compared with existing backup protection, e.g. Zone 2 of distance protection, the sensitivity of the scheme is relatively high (Zone 2 resistive reach in the UK is typically less than $50 \Omega$ [1]). In contract, the proposed scheme is capable of detecting high resistive faults (i.e. over $241 \Omega$ ). It should be noted that the proposed scheme is considered to provide an additional (economical) way of accelerating the vast majority of backup protection operations rather than complete substitution, and hence, improving system resilience and security.

The proposed scheme, which only requires voltage measurements at busbar levels, shows strong strengths in many aspects compared with current-based wide-area methods that require both voltage and current measurements.

1. The maximum fault resistance our scheme can detect is higher than many current-based schemes proposed in $[15,17]$.

2. Due to the accurate identification of faulted feeder, the scheme can operate relatively fast compared to impedance based methods proposed in [19].

3. Our scheme can report the status of protection devices to help the operators to make decision compared to other current-based schemes proposed in [15-19].

\subsection{Impact of communication latency}

Communication latency is a key issue to consider when evaluating the performance of schemes using wide area measurements. The level of communication latency is largely de- 


\begin{tabular}{c|c|c|c}
\hline Line & $R_{f 0 \max }(\Omega)$ & $\begin{array}{c}\text { Fault occurrence } \\
\text { identification time }(\mathrm{ms})\end{array}$ & $\begin{array}{c}\text { Faulted line } \\
\text { identification time (ms) }\end{array}$ \\
\hline 1 & 101 & 100 & 80 \\
\hline 2 & 201 & 100 & 80 \\
\hline 3 & 241 & 100 & 100 \\
\hline 4 & 241 & 80 & 80 \\
\hline 5 & 101 & 100 & 100 \\
\hline 6 & 241 & 100 & 100 \\
\hline 7 & 241 & 100 & 100 \\
\hline
\end{tabular}

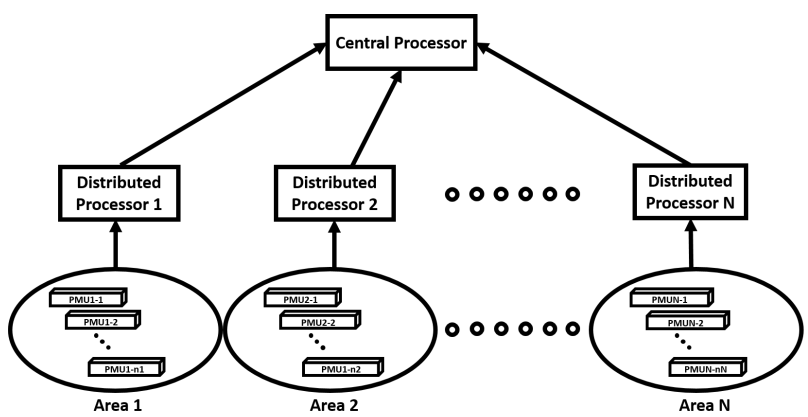

Table 4: Test results of single-phase to earth faults

Figure 14: Scheme structure for application in real networks

pendent on the infrastructure and technologies used and cobigskipuld lead to delays in the operation of this scheme. However, according to [30], the requirement for maximum point-topoint latency for wide-area monitoring and protection schemes is $35-55 \mathrm{~ms}$. Based on this specification, the impact of communication latency could lead to a maximum fault detection time of $135 \mathrm{~ms}$ and circuit breaker status analysis time of 235 $\mathrm{ms}$, which is still faster than conventional backup protection schemes, while retaining the benefits (selectivity, relative simplicity, utilizing existing PMUs, reduced number of IEDs, etc.).

In terms of data loss issues, Phasor Data Concentrators (PDCs) are typically used for processing and aggregating PMU data. There is extensive research has been conducted to handle such degraded communication performance for wide-area protection and control. Therefore, the data loss handling is not the focus of the paper. One of the most widely used methods is the interpretation of missing data, where the PMU data is buffered for a certain time period and the system will attempt to interpolate the missing data from the packet arriving before and after the missing packet $[31,32]$.

\section{Applicability to Large-Scale Systems}

In order to apply the scheme to an actual power system with potentially large number of nodes, a distributed wide-area hierarchy is proposed as shown in Fig. 14. Each area consists of several substations equipped with PMUs. The distributed processors handle the PMU data from their corresponding areas only. In the first instance, the distributed processor identifies whether the area includes a node with a voltage below the preset threshold. Subsequently, the node with the lowest voltage within the area is identified and noted, which is stage one of the scheme as explained in Section 2.2.

If more than one area indicates the presence of a fault, the central processor is then initiated to determine, through comparison, the smallest $k_{i}$ from the areas where voltages have fallen below the threshold. The node with the lowest voltage magnitude among these areas is assigned as $N_{\min }$ - the node with lowest $k_{i}$ in the network. If $N_{\min }$ is not a boundary node, the faulted circuit is identified within the corresponding area which includes $N_{\text {min }}$. The faulted region in this case includes $N_{\min }$ and all neighbouring nodes. Only the distributed processor corresponding to this area is required for further analysis, as only one area is involved and no information from other areas is required. The process follows the stages as outlined in Sections 2.3 (faulted circuit breaker identification) and 2.4 (backup protection). However, when $N_{\min }$ is a boundary node, the faulted circuit can be within either of the neighbouring areas as the faulted region includes $N_{\min }$ and all neighbouring nodes. in this case, multiple areas are involved, so the central processor is needed to analyse the information from all associated areas. With inputs from stage one applied to those areas, a combined faulted region is formed, which is then analysed by the central processor to identify the faulted circuit and protection failure (stage two), followed by the backup protection if required (stage three).

\section{Conclusions}

A PMU based wide-area backup protection scheme with a protection/circuit breaker performance analysis function has been presented in this paper, using only voltage magnitudes as input. The system identifies the presence of faults, identifies the faulted feeder, analyses protection performance, reporting on any failures of circuit breakers to operate and provides backup protection in case of any main protection/breaker failure. The presented scheme has been validated using the IEEE 14-bus system. It is shown that the system can effectively detect faults (including resistive faults) anywhere in the test system within $100 \mathrm{~ms}$. The subsequent faulted circuit identification and provision of information relating to protection/circuit breaker failure can be provided within a further $100 \mathrm{~ms}$. Backup protection function of the scheme (sending tripping signals to protection/circuit breakers) can be initiated immediately after faulted circuit identification and protection performance analysis. Additionally, a hierarchical decision making structure for application in large networks is proposed and explained. It has been demonstrated that the scheme can act as a fast, cost-effective wide-area backup protection and information/reporting system.

\section{References}

[1] National Grid, "Rte - transmission line protection," Tech. Rep., 2008.

[2] S. Horowitz and A. Phadke, "Third zone revisited," IEEE Trans. on Power Delivery, vol. 21, no. 1, pp. 23-29, Jan 2006.

[3] M. Ahsan, A. Chowdhury, S. Ahmed, et al., "Technique to develop auto load shedding and islanding scheme to prevent power system blackout," Power Systems, IEEE Trans. on, vol. 27, no. 1, pp. 198-205, Feb 2012. 
[4] S. Garlapati, H. Lin, A. Heier, S. K. Shukla, and J. Thorp, "A hierarchically distributed non-intrusive agent aided distance relaying protection scheme to supervise zone 3," International Journal of Electrical Power and Energy Systems, vol. 50, pp. 42 - 49, 2013.

[5] National Grid, "System Operability Framework 2016," Tech. Rep., 2016.

[6] Z. He, Z. Zhang, W. Chen, et al., "Wide-area backup protection algorithm based on fault component voltage distribution," IEEE Trans. on Power Delivery, vol. 26, no. 4, pp. 2752-2760, Oct 2011.

[7] D. Karlsson, M. Hemmingsson, and S. Lindahl, "Wide area system monitoring and control - terminology, phenomena, and solution implementation strategies," IEEE Power and Energy Magazine, vol. 2, no. 5, pp. 68-76, Sept 2004.

[8] "IEEE Standard for Synchrophasors for Power Systems," IEEE Std C37.118-2005 (Revision of IEEE Std 1344-1995), 2006.

[9] Vizimax. Synchrophaseur -pmu. [Online]. Available: https: //www.vizimax.com/products-services/phasor-measurement-unit

[10] J. Liao and C. He, "Wide-area monitoring protection and control of future power system networks," in IEEE Workshop on Advanced Research and Technology in Industry Applications, 2014, pp. 903-905.

[11] V. Terzija, "Adaptive underfrequency load shedding based on the magnitude of the disturbance estimation," Power Systems, IEEE Trans. on, vol. 21, no. 3, pp. 1260-1266, Aug 2006.

[12] A. Phadke, P. Wall, L. Ding, and V. Terzija, "Improving the performance of power system protection using wide area monitoring systems," Journal of Modern Power Systems and Clean Energy, vol. 4, no. 3, pp. 319-331, 2016.

[13] X. Liu, X. Zhang, and V. Venkatasubramanian, "Distributed voltage security monitoring in large power systems using synchrophasors," IEEE Trans. on Smart Grid, vol. 7, no. 2, pp. 982-991, March 2016.

[14] E. Udren, "Principles for Practical Wide-Area Backup Protection with Synchrophasor Communications," in CIGRE Paris Session, 2014.

[15] M. Neyestanaki and A. Ranjbar, "An adaptive pmu-based wide area backup protection scheme for power transmission lines," IEEE Trans. on Smart Grid, vol. 6, no. 3, pp. 1550-1559, 2015.

[16] M. Eissa, M. Masoud, and M. Elanwar, "A novel back up wide area protection technique for power transmission grids using phasor measurement unit," IEEE Trans. on Power Delivery, vol. 25, no. 1, pp. 270-278, 2010.

[17] S. Sheng, K. Li, W. Chan, X. Zeng, et al., "Adaptive agent-based widearea current differential protection system," IEEE Trans. on Industry Applications, vol. 46, no. 5, pp. 2111-2117, Sep 2010.

[18] J. Ma, C. Liu, and J. Thorp, "A wide-area backup protection algorithm based on distance protection fitting factor," IEEE Trans. on Power Delivery, vol. 31, no. 5, pp. 2196-2205, 2016.

[19] M. Chen, H. Wang, S. Shen, and B. He, "Research on a distance relaybased wide-area backup protection algorithm for transmission lines,' IEEE Trans. on Power Delivery, vol. 32, no. 1, pp. 97-105, 2017.

[20] Z. Fan, G. Song, C. Wang, and S. Bai, "Study on distance protection based on wide area information," in China International Conference on Electricity Distribution (CICED), 2016, pp. 1-6.

[21] J. Zare, F. Aminifar, and M. Sanaye-Pasand, "Synchrophasor-based widearea backup protection scheme with data requirement analysis," IEEE Trans. on Power Delivery, vol. 30, no. 3, Jun 2015.

[22] J. Tan, P. Crossley, P. McLaren, P. Gale, I. Hall, and J. Farrell, "Application of a wide area backup protection expert system to prevent cascading outages," IEEE Trans. on Power Delivery, vol. 17, no. 2, pp. 375-380, Apr 2002.

[23] S.-S. Mirhosseini and M. Akhbari, "Wide area backup protection algorithm for transmission lines based on fault component complex power," International Journal of Electrical Power and Energy Systems, vol. 83, pp. $1-6,2016$.

[24] E. O. Schweitzer and S. E. Zocholl, "Introduction to symmetrical components," 2011.

[25] A. J. Roscoe, I. F. Abdulhadi, and G. M. Burt, "P and m class phasor measurement unit algorithms using adaptive cascaded filters," IEEE Transactions on Power Delivery, vol. 28, no. 3, pp. 1447-1459, July 2013.

[26] A. J. Roscoe, "Exploring the relative performance of frequency-tracking and fixed-filter phasor measurement unit algorithms under c37.118 test procedures, the effects of interharmonics, and initial attempts at merging p-class response with m-class filtering," IEEE Transactions on Instrumentation and Measurement, vol. 62, no. 8, pp. 2140-2153, Aug 2013.

[27] A. J. Roscoe, I. F. Abdulhadi, and G. M. Burt, "P and m class phasor mea- surement unit algorithms using adaptive cascaded filters," IEEE Transactions on Power Delivery, vol. 28, no. 3, pp. 1447-1459, July 2013.

[28] ABB, "Instrument transformers - technical information and application guide."

[29] A. Amberg and A. Rangel, "Tutorial on symmetrical components," 2013.

[30] CIGRE Working Group C4.34, "Application of Phasor Measurement Units for Monitoring Power system Dynamic Performance, September 2017,” Tech. Rep., 2014.

[31] R. Pourramezan, Y. Seyedi, H. Karimi, G. Zhu, and M. Mont-Briant, "Design of an advanced phasor data concentrator for monitoring of distributed energy resources in smart microgrids," IEEE Transactions on Industrial Informatics, vol. 13, no. 6, pp. 3027-3036, Dec 2017.

[32] M. Kanabar, M. G. Adamiak, and J. Rodrigues, "Optimizing wide area measurement system architectures with advancements in phasor data concentrators (pdcs)," in 2013 IEEE Power Energy Society General Meeting, July 2013, pp. 1-5. 